\title{
An Automated Probe Handling and Image Inspection System for the Recognition of Biological Hazardous Material in the Air
}

\author{
Petra Perner
}

\begin{abstract}
Human beings are exposed every day to bio-aerosols in their personal and professional life. The European Commission has issued regulations for protecting employees in the workplace from biological hazards. Airborne fungi can be detected and identified by an image-acquisition and interpretation system. In this paper, we present recent results on the development of an automated image acquisition, sample handling and image-interpretation system for airborne fungi identification. We explained the application domain and described the development issues. The development strategy and the architecture of the system are described, and results are presented.
\end{abstract}

Index Terms-Health monitoring, Microscopic image acquisition, microbiological sample handling, image analysis, image interpretation, case-based object recognition, case-based reasoning.

\section{INTRODUCTION}

Airborne microorganisms are ubiquitously present in various indoor and outdoor environments. The potential implication of fungal contaminants in bio-aerosols on occupational health has been recognized as a problem in several working environments. The exposure of workers to bio-aerosols is a concern, especially in composting facilities, in agriculture, and in municipal waste treatment. The European Commission has therefore issued guidelines protecting employees in the workplace from airborne biological hazards. In fact, the number of incidents of building-related sickness, especially in offices and residential buildings, is increasing. Some of these problems are attributed to biological agents, especially to airborne fungal spores. However, the knowledge of health effects of indoor fungal contaminants is still limited. One of the reasons for this limitation is that appropriate methods for rapid and long-term monitoring of airborne microorganisms are not available.

In addition to the detection of parameters relevant to occupational and public health, in many controlled environments, the number of airborne microorganisms must be kept below the permissible or recommended values, e.g. in clean rooms, in operating theatres, and in domains of the food

Manuscript received January 10, 2018; revised March 23, 2018. This work was sponsored under the High-Tech Strategy of the Federal Republic of Germany under the grant number 16IN0147 entitled "The Development of Novel Image Analysis and Interpretation Methods for Airborne Hazardous Materials - BIOGEFA". We appreciate the great financial support that allowed us to do and explore substantial work on the subject.

Petra Perner is with the Institute of Computer Vision and applied Computer Sciences, PF 3011 14, 04251 Leipzig, Germany (e-mail: pperner@ibai-institut.de). and pharmaceutical industry. Consequently, the continuous monitoring of airborne biological agents is a necessity for the detection of risks to human health as well as for the flawless operation of technological processes.

At present, a variety of methods are used for the detection of fungal spores. The culture-based methods depend on the growth of spores on an agar plate and on the counting of colony-forming units [1].

Culture-independent methods are based on the enumeration of spores under a microscope, the use of a polymerase chain reaction or on DNA hybridization for the detection of fungi [1]. However, all these methods are limited by time-consuming procedures of sample preparation in the laboratory. This paper describes the development and the realization of an automated image-acquisition and sample handling unit of biologically dangerous substances and the automated analysis and interpretation of microscope images of these substances.

In the system described here, contaminated air containing bio-aerosols are collected in a defined volume via a carrier agent. Bio-aerosols are recorded by an image-acquisition unit, counted, and classified. Their nature is determined by means of an automated image-analysis and interpretation system. Air samples are automatically acquired, prepared and transferred by a multi-axis servo-system to an image-acquisition unit comprised of a standard optical microscope with a digital colour camera.

In Section II we give general remarks to the image analysis of microorganism. Related work is described in Section III. Then we described development issues in Section IV and system requirements in Section V. The automated probe handling and imaging unit is described in Section VI.

The variability of the biological objects is very broad. Given the constraints of the image acquisition, this variability is found in the appearance of the objects as well. There are no general features allowing one to discern the type of the detected fungi. In the system employed here, images are stored, and a more generalized description of the different appearances of the same objects is used. We will describe this novel case-based reasoning approach of the image analysis and its interpretation in Section VII. Finally, we will summarize our work in Section VIII.

\section{General Comments on the IMAGE ANALYsis of MICROORGANISM}

Classification of airborne fungal spores from environmental samples present the image analyst with 
inherent difficulties. Most of these difficulties concern the automatic identification of microorganism in general [2]. For example, the types and numbers of objects (different fungal species) that may be present in any one air-sample are both unknown and effectively unlimited. Also, intraspecies variation of characteristics (such as size, color or texture of spores) can be large and may depend on several factors. Furthermore, the bulk size of two targeted species may be an order of magnitude or more apart, making it difficult to decide e.g. on an optical magnification setting. The dynamic and variable nature of the microorganism thus presents a formidable challenge regarding the design of a robust image interpretation system with the ideal characteristics of high analysis accuracy but wide generalization ability. The difficulties can be summarized as follows:

- Intraspecies variation due to natural phenomenon, i.e., life-cycle, environmental effects

The dynamic nature of living organisms results in properties such as size or color of the microorganism being statistically variable. Different growth condition of microorganism may result in uncharacteristically large or small specimens - resulting in data outliers. Ultimately, under these circumstances, the classification accuracy of an image interpretation system will rely on the training database capturing as much of this variability as possible.

- Intraspecies variation due to predation, fragmentation etc.

Often atypical characteristics occur due to predation, environmental factors, or aging.

- To stain or not to stain?

Many species appear clear/opaque at the resolutions used, making imaging and analysis very difficult. Staining can help to increase the resolution of the fungal material and to distinguish between viable and non-viable organisms. Depending on the application, different stains must be used. At present 10-20 different stains are frequently used for staining fungal spores. They include "all-purpose"-stains such as lactophenol cotton blue, which stains fungal elements blue. The staining procedure takes only 1 to 2 minutes. The application of fluorescence stains allows discriminating between living and dead cells. However, the use of epifluorescence microscopy in an automated system is more expensive and requires additional hardware. While it is common to stain specimen samples prior to analysis, staining puts special demands on an automated sample handling, image acquisition system and image interpretation system.

- Choosing an appropriate optical resolution for imaging

The wide variation of the size of targeted species necessitates a choice of optical magnification that may not be optimal for any species. For example, to analyze the fine internal structures of species such as Wallemia sebi, a 1000x magnification would be required. Fusarium spores are the largest spores among the spores considered in this study. They would require only a 200x magnification instead of a $1000 \mathrm{x}$ magnification.

\section{- Imaging 3-dimensional objects}

A spore is a 3-dimensional object. Imagine a spore which has an ellipsoid shape. Depending on its position, the object can appear as a round object or as an elongated object in a 2-D image. Many species have a significant length in the third dimension, often greater than the depth-of-field of the imaging device, making their representation as a 2-D image difficult. As such, significant areas of the specimen will be out of focus. If only one kind of specimen appears in an image, focusing may not be so difficult. However, in a real air sample, different specimen can appear. In this case, a single focus level may not be sufficient. Various levels of focus may be necessary which will result in more than one digital image for one sample.

- How to get a clean sample from the air sample?

Samples of bioaerosols will contain a wide range of objects (organic and inorganic particles). Filters will be needed to remove particles larger than the objects of interest. But this will generally not prevent the image from containing non-targeted species. Non-targeted species/objects will generally needed to be classified. Normally, the should be covered by water and a cover glass. To realize this in an automated handling system is not easy since handling glass by means of handling devices is difficult.

\section{RELATED WORKS}

Several case studies have been done on identifying fungi or other microorganisms. In [3], an image analysis method was described for the identification of colonies of nine different Penicillium species as seen after growth on a standard medium. In [4], a study of image analysis based on fluorescence microscopy images was described for the improvement of the exposure assessment of airborne microorganism. Semiautomatic image analysis techniques were applied to segment the contour of fungal hyphae in [5]. Yeast cells were analyzed by image analysis techniques in [6]. Different Fusarium species macroconidia were analyzed in [7]. The work aimed at designing an automated procedure for collecting and documenting microscopic pictures of Fusarium conidia, determining various morphological parameters and statistically evaluating the effectiveness of those characteristics in differentiating the most important pathogenic Fusarium species occurring on wheat in Germany.

The work which is most closely related to our work is that described in [8]. The ability of an image analysis routine to differentiate between spores of eleven allergenic fungal genera was tested using image analysis based on seven basic and up to 17 more complex features, extracted from digitized images. Fungal spores of Alternaria, Cladosporium, Fusarium, Aspergillus, Botrytis, Penicillium, Epicoccum, Exserohilum, Ustilago, Coprinus and Psilocybe were examined in a series of experiments designed to differentiate between spores at the genus and species level. No specific algorithm for image enhancement and image segmentation is described in this work. It appears that only the feature measurement had been automated. The object area was labelled interactively. From the fungal spores, seven basic features including length, width, width/length ratio, area, form factor (circularity), perimeter and roundness, and 17 more complex features including equivalent circular diameter, compactness, box area, radius, modification ratio, sphericity, convex hull area, convex hull perimeter, solidity, concavity, 
convexity, fiber length, fiber width were extracted. Linear and quadratic discriminant analyses were used for classification. It is interesting to note that the authors created a sufficiently large database of fungi spores for their analysis. The number of spores used in this study ranges from 200 to 1000 samples. The classification accuracy according to a particular class ranged from $56 \%$ to $93 \%$ for general comparison and from $26 \%$ to $97 \%$ for species comparison. The results showed that not for all classes the right features for classification were selected. Rather, it appeared that all common features that are known in pattern recognition for the description of a 2-D objects were applied to the images. No specific features have been developed that described the properties of the different fungi genera and species. For example, considering species Fusarium, the septation is a highly discriminating feature but no such description was included in the feature list [8].

Many successful case studies have been conducted to automate the identification of fungi and microorganism in general. In these studies, imaging methods for microorganism, automatic focusing methods, image analysis, feature description and classification have been developed. Most of these studies used 500x to 1,500x magnification for image acquisition. The most used feature descriptors are the area size and the shape factor of circularity. The colour information was used only in [3], and was neglected in all other studies. Not all publications included microscopic images of the microorganism; therefore, we cannot evaluate the quality of the images. In most of the cases, the digitized images were not highly structured. The objects and the background appeared homogenous allowing to apply a simple thresholding technique for image segmentation. In general, these studies are characterized by applying standard image analysis and feature extraction procedures to the images. Neither a specific feature set for fungi identification has been developed nor a good feature set for the description of microorganism has been found yet as evidenced by [7] and [8].

The difference to our work is that in most of these studies, images are created for only one species and not for a variety of distinct species, except for the work in [8]. The creation of digitized images for a variety of distinct species is much harder since the species differ in size and dimension and, therefore, the selection of an optical resolution that will show the image details of the different species in sufficient resolution is not easy. Also, the image analysis is much more difficult since for all the different objects a sufficient image quality should be reached after image segmentation.

\section{DEVELOPMENT ISSUES}

We decided to start the development of our system based on a data set of fungi spore images taken in the laboratory under optimal conditions and constant climate conditions. The data set should represent the prototypical appearance of the different kind of fungi strains and serve as a gold standard.

The objects in the images are good representatives of the various kinds of fungal spores cultured under optimal conditions and constant climate conditions. However, as it can be seen from the images of Alternaria alternata and
Ulocladium botrytis that none of the objects in the image looks like another. There is no clear prototypical object. We can see a high biological variability and younger and older representatives of the fungal strains. Depending on the image acquisition conditions we see objects from the side and from the top and this influences the appearance of the objects. Generalization about the objects cannot be done manually, rather, each case that appears in practice should be stored in the system and the system should learn more generalized descriptions of the different appearance of the same objects over time. All this suggests that a case-based reasoning approach for the image interpretation [9] should be taken rather than a generalized approach. Case-Based Reasoning [10] is used when generalized knowledge is lacking. The method works on a set of cases previously processed and stored in a case base. A new case is interpreted by searching for similar cases in the case base. Among this set of similar cases, the closest case with its associated result is selected and presented on a display.

For the kind of images created in the laboratory, we had to develop an image analysis procedure. It is then necessary to describe the images by image features and to develop a feature extraction procedure which can automatically extract the features from the images. The features and the feature values extracted from the images together with the name of the fungal spores make up an initial description of the data. We do not know if all image features are indeed necessary. However, we extracted as many image features as possible from the images that appear meaningful in some way to ensure that we can mine the right case description from the database. From this initial description of the data, we need to identify good representative descriptions for the cases by using case mining methods [10]. Based on this information we generated the case-based reasoning system.

After reaching a sufficient classification accuracy, we started to include real air samples into the system by adapting the prototypical representations of fungi spores to the real ones.

\section{SYSTEM REQUIREMENTS}

The system to be developed should allow to collect dust and biological aerosols in well-defined volumes over microscope slides, deposit them there, image them with an appropriate method, count and classify them with an automated image analysis and interpretation method, to determine the following parameters from the images:

- Total number of airborne particles

- Classification of all particles according to their size and shape

- Classification of biological particles according to their size and shape, e.g. spores, fragments of fungal mycelia, and fragments of insects

- Number of respirable particles

- Total number of airborne particles of biological origin

- Number of dead particles of biological origin

- Number of viable and augmentable particles of biological origin

- Identification of species or genera exploiting the characteristic shapes of spores and pollen 
- Proportion of airborne abiotic and biotic particles

- The proportion of dead and viable airborne microorganisms.

At the beginning of the project, the following requirements concerning the optical and the mechanical system were defined:

- Colour images should be produced to facilitate the separation of dead and living objects.

- It should be possible to generate images in at least three defined depths of field.

- A marker liquid like lactophenol should be used to further enhance the separation of dead and living objects (blue colour for living objects). For this purpose, a cover slip is necessary to uniformly distribute the marker drop on the object slide.

- The object slide should be covered with an adhesive to fix the airborne germs.

Six fungal strains representing species with different spore types were identified as important species in different environments (Tab. I) By our industrial project partner JenaBios $\mathrm{GmbH}$. A database of images from the spores of these species was produced and was the basis of our development. The number of imaged spore per species was about 30-50. Since no commercial system was known fulfilling all requirements, a corresponding system was developed which is described as follows.

\begin{tabular}{lllll}
\multicolumn{4}{c}{ TABLE I. STRAINS OF EMPLOYED FUNGI AND SELECTED PROPERTIES OF SPORES } \\
\hline Species & Strain no. & Spore shape & Spore colour & Spore size $[\mu \mathrm{m}]$ \\
\hline Alternaria alternata & $\mathrm{J} 37\left(\mathrm{~A}^{1}\right)$ & septated, clavate to ellipsoidal & pale brown & $18-83 \times 7-18$ \\
Aspergillus niger & $\mathrm{i} 400\left(\mathrm{~B}^{2}\right)$ & spherical, ornamented with warts and spines & brown & $\emptyset 3.5-5$ \\
Rhizopus stolonifer & $\mathrm{J} 07(\mathrm{~A})$ & irregular in shape, often ovoid to elliptical, striate & pale brown & $7-15 \times 6-8$ \\
Scopulariopsis brevicaulis & $\mathrm{J} 26(\mathrm{~A})$ & spherical to ovoid & rose-brown & $5-8 \times 5-7$ \\
Ulocladium botrytis & $\mathrm{i} 171(\mathrm{~B})$ & septated, ellipsoidal & olive-brown & $18-38 \times 11-20$ \\
Wallemia sebi & $\mathrm{J} 35(\mathrm{~A})$ & cubic to globose & pale-brown & $\varnothing 2.5-3.5$ \\
\hline
\end{tabular}

1(A): from culture collection of JenaBios GmbH, Jena, Germany

2(B): from the fungal stock collection of the Institute of Microbiology, University of Jena, Jena, Germany

\section{The Automated Probe Hanling AND IMAgING SYSTEM}

\section{A. The Microscopic Image-Acquisition System}

Following the specifications given in Section II, we developed an automated sample-handling and digital image-acquisition system for taking microbiological material from air samples. An existing optical Leitz microscope was upgraded and its hardware expanded. A lens from Olympus with a magnification of $60 \mathrm{X}$ and a numerical aperture of 0.7 was used. Its focal length of $1.7 \mathrm{~mm}$ provided sufficient clearance between the lens and the object slide including the cover glass to avoid collisions due to their variability in thickness. The lens was inserted in an autofocusing device from Physik Instrumente (PI, Karlsruhe, Germany) which was mounted on the lens revolver. A motorized xy-table from Märzhäuser (Wetzlar, Germany) with a controller was used to arbitrarily shift the object slide in both $\mathrm{x}$ and $\mathrm{y}$-direction. For the digital image acquisition, a 1.4 Mpixel colour digital camera from Soft Imaging System (SIS, Münster, Germany) was used. Our estimates showed that a pixel number larger than 1.4 Mpixel is sufficient for the given magnification. Fig. 1 demonstrates that the optical resolution is sufficient to recognize details in spores like Ulocladium.

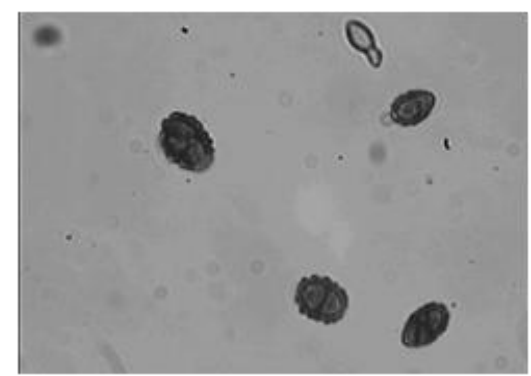

Fig. 1. Image demonstrating the resolution of the optical microscope used. The microscopical image displays spores of Ulocladium. The field of view is $134 \times 100 \mu \mathrm{m}^{2}$. The sample was prepared by AUA/JenaBios, lens Olympus $60 \mathrm{X} / 0.70$. The resolution of this image is $5 \mu \mathrm{m}$.
The functions of image acquisition and image storage, movement of the specimen in $\mathrm{x}$ and $\mathrm{y}$-direction, and auto-focusing in the $\mathrm{z}$-direction are controlled by the AnalySIS Pro software from SIS. A pattern of images at any image position can be freely programmed and stored in a macro-code. This holds true also for the number of images to be captured. If necessary, it is possible to automatically capture images at different depths of focus around the optimum position. By the automatic shading correction, the effect of an inhomogeneous illumination of the object can be removed.

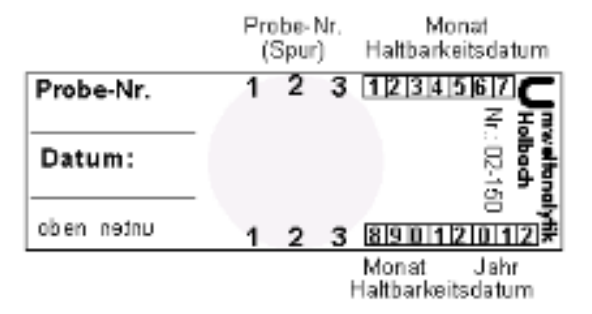

Fig. 2. Object slide of standard size $76 \times 26 \times 1 \mathrm{~mm}^{3}$ with a central sticky layer [11]; Image obtained from Umweltanalytik Holbach.

\section{B. The Automatic Sample-Acquisition and Handling System}

The following chapter describes the main units and functions of the demonstration set-up realized during the project. A stock of special object slides covered with a sticky layer and obtained from Umweltanalytik Holbach [11], (Fig. 2 ) is kept in a slide storage. A sliding gripper takes the lowest slide in the storage and transports it into the slit impactor obtained from Umweltanalytik Holbach (Fig. 3). The object slides are separated by distance holders with a corresponding recess, to avoid sticking between the slides. The distance holder is removed by the same gripper, now moving in the opposite direction and depositing the distance holder into a box. The distance holders can be used again when the slide deposit is reloaded.

In the slit impactor (Fig. 3), the air, potentially containing 
airborne germs, is guided into the sticky area of the object slide by the air stream generated by an air pump. After a few tens of seconds adjusted appropriately, the pump is switched off and the object slide is transported to the pipetting unit driven by the dosing pump (Cavro XL 3000 obtained from Tecan Systems San Jose, Ca, USA). To achieve this, the object slide must change its transporting axis and thus its direction of movement. From a thin nozzle, one drop of lactophenol is deposited on the sticky area of the object slide. The object slide is afterwards transported through the coordinate origin to the cover-slip gripper unit. This gripper acts as a low-pressure sucker and takes one cover glass from the deposit and places it with one edge first on the object slide. Then the cover glass can drop, down on the object slide and flattens the drop so that it will be distributed all over the sticky area forming a thin layer. In this way, the airborne germs collected on the sticky layer are immersed in the lactophenol. In lactophenol, living germs take on a blue colour. The object slide is then transported back to the coordinate origin where it again changes its direction of movement by $90^{\circ}$ and is transported to the xy-table of the microscope where the slide is received and directly transported into a position underneath the lens. To this end, an additional module was integrated into the AnalySIS Pro software. It controls the manual or automated shift of the xy-table between the image-acquisition position under the lens and the loading position, where the object slide is shifted from the object-slide preparation unit to the xy-table. After the object slide has reached the image acquisition position, the microscope camera then takes the images at the programmed slide positions after auto-focusing of the microscope lens at each position. The cycle of shifting the xy-table of the defined positions, auto focusing, image acquisition, and storage is programmable in a macro-code integrated into the AnalySis Pro software. This can also be done for other procedures like shading correction or image acquisition at different z-positions. After finishing the imaging sequence, the slide is transported away from the xy-table with a special arm and dropped into a box. While the image grabbing procedure by the microscope unit is still underway, the object-slide preparation unit already starts with the preparation of a new object slide. The object-slide preparation and manipulation are performed by a hardware controller and by custom software written in $\mathrm{C}++$. The transfer from the AnalySIS Pro software to the $\mathrm{C}++$ software and vice versa is controlled by a communication protocol as an interface between both software units. Altogether six different mechanical axes must be handled, not counting the axes of the xy-table (Fig. 4). The unit for object-slide preparation and the expanded microscope are shown in Fig. 5a and Fig. 5b.

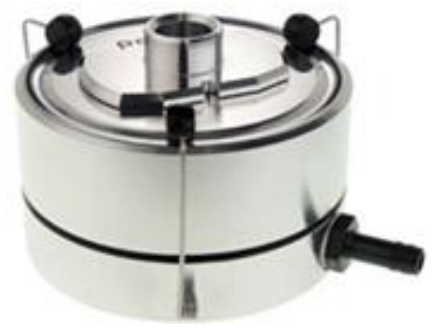

Fig. 3. Slit impactor for collection of airborne particles [11]; Image obtained from Umweltanalytik Holbach.

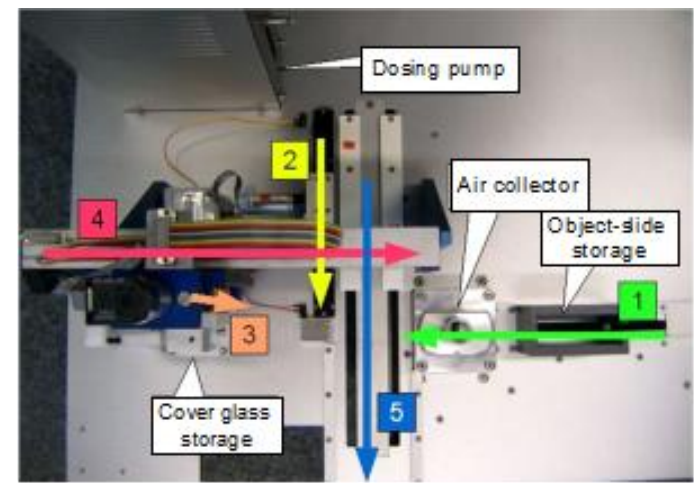

Fig. 4. Top view of the mechanical unit for moving object slides, indicating also the position of the cover-glass storage, the dosing pump for lactophenol, the slit impactor or air collector, and the storage for the object slides. The numerals $1-5$ indicate the sequences of the movements; axis No. 6 is not shown.

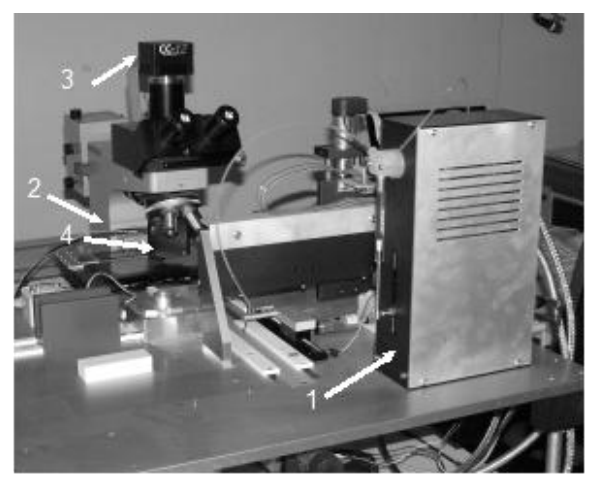

Fig. 5a. Prototype set-up showing the dosing pump (arrow 1), several axes, the optical microscope with xy-table (arrow 2), and the digital camera

(CC-12, arrow 3). The auto-focusing unit holds the lens (arrow 4).

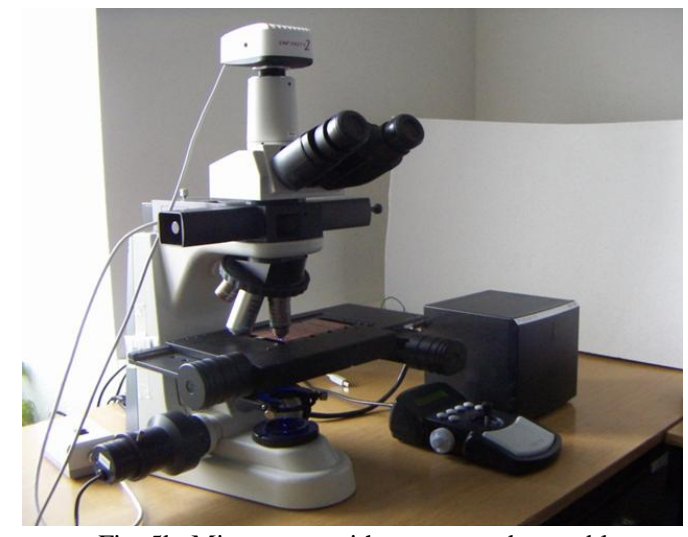

Fig. 5b. Microscope with camera and x-y table.

\section{IMAGE ANALYSIS}

Once an image has been taken it transferred to the image-analysis unit for further processing. We will describe the overall architecture of the system [13] and its single components in the next sections.

\section{A. The Architecture}

The architecture of the system is shown in Figure 6. Objects are recognized in the microscopic image by a case-based object-recognition unit [14]. This unit has a case-base of shapes (case base_1) for fungi spores and determines on a similarity-based inference if there are objects in the image that have a similar shape as the ones stored in the case base. In this case, the objects are labelled and transferred for further processing to the feature-extraction unit. To ensure proper 
performance of this unit, the general appearance of the shapes of the fungi spores must be learned. To this end, we have developed a semi-automated procedure [14] that allows acquisition of the shape information from the raw image data and learning of groups of shape-cases and general shape-cases A more detailed description of the case-based object-matching unit can be found in Section VII $B$.

The feature-extraction procedures are based on the knowledge of an expert. Note that a particular application requires special feature descriptors. Therefore, not all possible feature-extraction procedures can be implemented in such a system from the beginning. Our aim was to develop a special vocabulary and the associated feature-extraction procedures for application in fungi identification, as described in Section VII $C$.

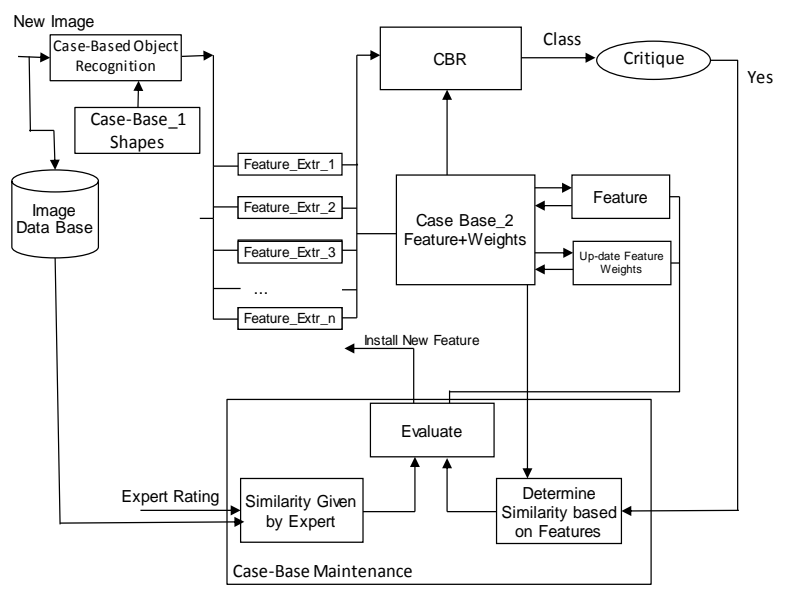

Fig. 6. System architecture.

Suppose that fungi species are wrongly identified by the system. Then a case-based maintenance process will start. First the system developer must check whether new features must be acquired for each case, or whether the whole case representation should be updated based on the learning procedures. The feature weights are learnt, as well as a subset of relevant features (see Section VII D). To acquire new features means that necessary feature-extraction procedures must be developed and that of all cases the new features must be calculated and inputted into the existing case description. Therefore, the digital images acquired so far are retained in the image-database. Then, the case representation, as well as the index structure, must be updated. This ensures that we can generate step-by-step a system that can describe the variability of the different biological objects that may appear.

\section{B. Case-Based Object Recognition}

The objects in the image are highly structured. Our study has shown that the images specified in Fig. 8 cannot be segmented by thresholding. The objects in the image may be occluded, touching, or overlapping. It can also happen that only part of the object appears in the image. Therefore, we decided to use a case-based object recognition procedure [14] for the detection of objects in the image.

A case-based object-recognition method uses cases that generalize the original objects and compares them with the objects of the image (Fig. 7). During this procedure, a score is calculated that describes the quality of the fit between the object and the case. The case can be an object model which describes the inner appearance of the object as well as its contour. In our case, the appearance of the objects can be very diverse. The shape seems to be the feature that generalizes the objects. Therefore, we decided to use contour models. We do not use the grey values of the model, but instead the object's edges. In determining the score of the match between the contour of the object and the case, we use a similarity measure based on the scalar product that measures the average angle between the vectors of the template and the object.

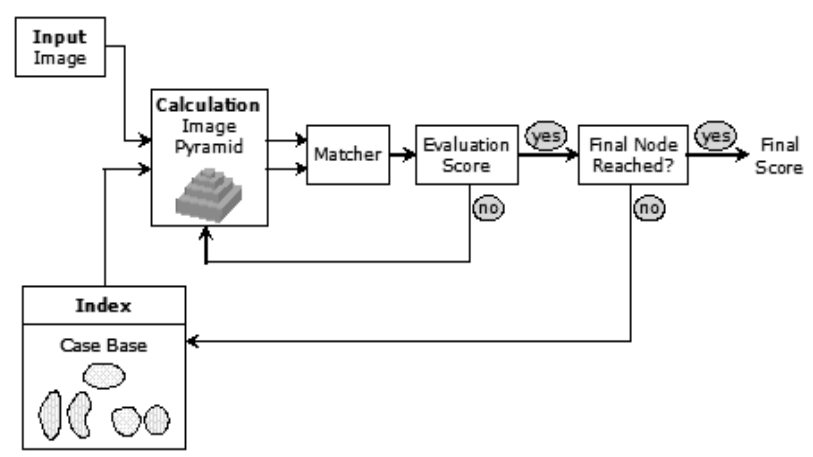

Fig. 7. The principle of case-based object-recognition architecture.

\section{Case-Base Generation}

The acquisition of the case is done semi-automatically. Prototypical images were shown to an expert. The expert manually traced the contour of the object by means of the cursor on the computer. Afterwards, the number of contour points is reduced for data-reduction purposes by interpolating the marked contour by a first-order polynomial. The marked object shapes are then aligned by the Procrustes Algorithm [15]. From the sample points, the direction vector is calculated. From a set of shapes, general groups of shapes are learnt by conceptual clustering, which is a hierarchical incremental clustering method [16]. The prototype of each cluster is calculated by estimating the mean shape [16] of the set of shapes in the cluster and is taken as a case model.

\section{Results for Case-Based Object Recognition}

We had a total of 10 images for each class at our disposal. From this set of images, two images were selected for case generation. In these two images, there were approx. 60 objects. These objects were labelled and used for the case generation according to the procedure as described in Section VII $C$. The result was a database of cases. These cases were applied to the image for the particular class.

The threshold for the score was set to 0.8 . We calculated the recognition rate as the number of objects that were recognized in the image to the total number of objects in the images. Note that the recognition rate can be higher than $100 \%$ since our matching procedure also fires in image regions where no objects are present due to background noise. The aim is to configure the case-based object-recognition unit in such a way that the number of false alarms is low. The results of the matching process are shown in Figs. 8 and 9. The highest recognition rate can be achieved for the objects Aspergillus niger and Scopularioupsi since the shape of these objects does not vary much. This is also expressed by the number of models, see Table II. These classes have the lowest number of cases. For those classes where the variation of the 
shape of the objects is high, the number of the cases is also high. The recognition rate shows that we did not have enough cases to recognize the classes with a good recognition rate (see Ulocladium botrytis and Alternaria alternata). Therefore, we needed to increase the number of cases. For this task, we developed an incremental procedure for the case acquisition in our tool. Objects that had not been recognized well were displayed automatically for tracing and then the similarity to all other shapes were calculated. The clustering was done in an incremental fashion as well [16]. This procedure will ensure that we can learn the natural variation of the shape during the usage of the system.

\section{E. Case Description and Feature Extraction}

We chose an attribute-value pair-representation of the case description. The case consisted of the solution, i.e., the type of fungi spores and the features describing the visual properties of the object (see Fig. 9). From each recognized object a set of features was extracted. One feature is the case number which represents the shape of the object, the similarity score between the actual shape and the shape in the case base, the size of the object, various grey-scale features, and the texture inside the object. For the description of the texture, we used our texture descriptor based on random sets described in [17].
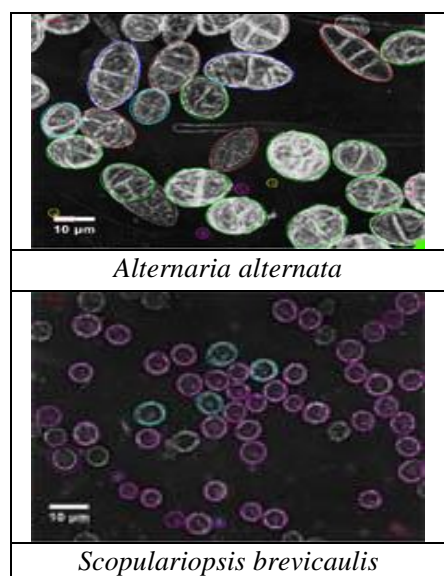

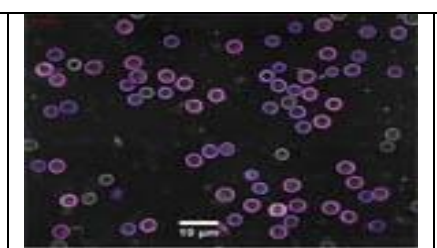
Aspergillus niger

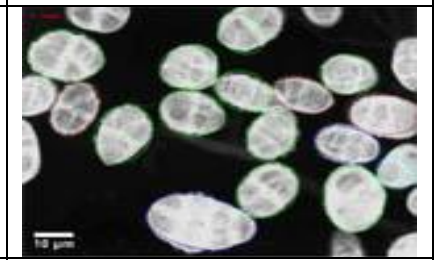

Ulocladium botrytis

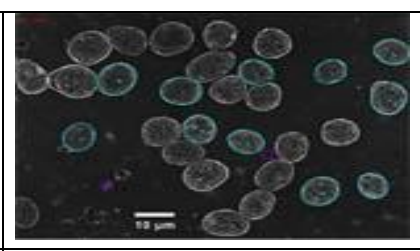
Rhizopus stolonifer

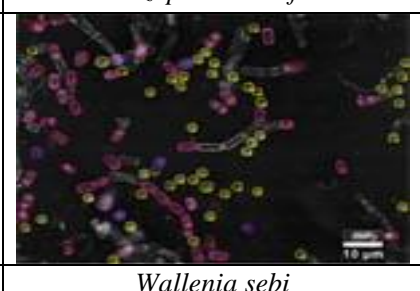

Wallenia sebi

Fig. 8. Recognized objects in the image.

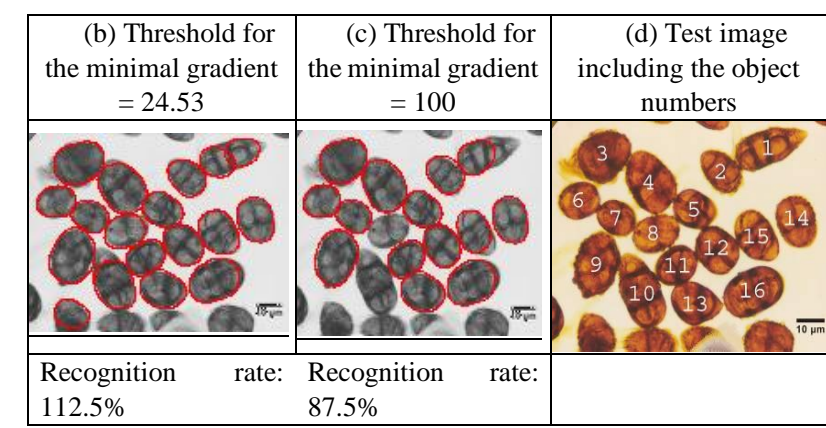

Fig. 9. Comparison of the matched objects by applying different thresholds for the minimal gradient.

TABLE II: RESULTS OF MATCHING

\begin{tabular}{|c|c|c|}
\hline Classes & $\begin{array}{c}\text { Number of } \\
\text { models }\end{array}$ & Recognition rate \\
\hline Alternaria alternata & 34 & 65.9 \\
\hline Aspergillus niger & 5 & 95.2 \\
\hline Rhizopus stolonifer & 22 & 87.7 \\
\hline Scopularioupsi & 8 & 94.5 \\
\hline Ulocladium botrytis & 30 & 77.2 \\
\hline Wallenia sebi & 10 & 90.3 \\
\hline
\end{tabular}

\section{F. References}

Our case-based reasoning procedure to recognize spores relies on prototype-based classification schemes [21]. Usually, such schemes are generalized from a set of single cases. Here, we have prototypical cases represented as images that were selected by humans. This means that, when building our system, we started from the top and had to collect more information about the specific class during usage of the system. Since a human has selected the prototypical images, his decision on the importance of an image might be biased; moreover, selecting only one image might be difficult for a human. He might have stored more than one image as prototypical images. Therefore, we needed to check the redundancy of the many prototypes for one class before taking them all into the case base. According to this consideration, our system must fulfill the following functions:

- Classification based on the nearest neighbor rule

- Prototype selection by a redundancy-reduction algorithm; feature weighting to determine the importance of the features for the prototypes

- Feature-subset to select the relevant features from the complete set of the respective domain.

The classification method is based on the nearest-neighbor rule. Since the prototypes are available at the same time, we chose a decremental redundancy-reduction algorithm proposed by Chang [18] that deletes prototypes if the classification accuracy does not decrease. The feature-subset selection is based on the wrapper approach [19] and an empirical feature-weighting learning method [20] is used. Furthermore, cross-validation is used to estimate the classification accuracy. The prototype selection, the feature selection, and the feature-weighting steps are performed during each run of the cross-validation process. This rule classifies in the category of its nearest neighbor [21]. More precisely, we call a nearest neighbor to if, where $i=1,2, \ldots n$. The nearest neighbor rule classifies into category $\mathrm{Cn}$ where is the nearest neighbor to and belongs to class $\mathrm{Cn}$. For the $\mathrm{k}$-nearest neighbor, we require k-samples of the same class to satisfy the decision rule. As a distance measure, we used the Euclidean distance. The recognition rate was evaluated on a database of 50 samples for each class based on cross-validation. The result is shown in Table III. Based on this result, we can conclude that the classification accuracy is 
higher than the recognition rate for some classes. This means that it is more difficult to recognize the objects that are most likely to be fungi spores than to classify them based on the extracted features.

.In the display of the system, the operator will find the acquired image in one window and in the other window the determined fungi spores and their total number. The system called Fungi PAD correctly identified the name of the fungi spores and their number.

TABLE III: CLASSIFICATION ACCURACY

\begin{tabular}{|l|c|}
\hline Classes & Classification accuracy \\
\hline Alternaria alternata & 90.4 \\
\hline Aspergillus niger & 95.0 \\
\hline Rhizopus stolonifer & 92.0 \\
\hline Scopularioupsi & 96.0 \\
\hline Ulocladium botrytis & 94.0 \\
\hline Wallenia sebi & 92.0 \\
\hline
\end{tabular}

\section{CONCLUSIONS}

In this paper, a system for an automated image acquisition and analysis of hazardous biological material in the air is described. It consists of an image-acquisition unit, its sample-handling hardware, and the image-interpretation system. The sample-handling and image-acquisition unit collects the airborne germs, deposits them on an object slide, disperses them with a marker fluid, and takes digital images of the germs in a programmable pattern. The stored images are analysed in order to identify the germs based on a novel case-based object-recognition method. The case generation is done semi-automatically by manually tracing the contour of the object, by automated shape alignment and by shape clustering, and eventually by prototype calculation. Based on the acquired shape cases, the object-recognition unit identifies objects in the image that are likely to be fungi spores. The further examination of labelled objects is done by calculating more distinct object features, from which a prototype-based classifier determines the kind of fungi spores. After all, objects had been classified by their type, the number of one type of fungi spores is calculated and displayed for the operator on the computer screen.

The recognition rate is good enough for on-line monitoring of environments. The final information can be used to determine the contamination of environments with biological hazardous material. It can be used for health monitoring as well as for process control.

\section{REFERENCES}

[1] W. Mücke and C. Lemmen, Schimmelpilze-Vorkommen, Gesundheitsgefahren, Schutzmaßnahmen, Ecomed-Verlag, Landsberg, 2004.

[2] F. H. L. Benyon, A. S. Jones, E. R. Tovey, and G. Stone, "Differentiation of allergenic fungal spores by image analysis, with application to aerobiological counts," Aerobiologia, vol. 15, pp. 211-223, 1999.

[3] R. F. Walker and M. Kumagai, "Image analysis as a tool for quantitative phycology - a computational approach to cyanobacterial taxa identification," Limnology, vol. 1, no. 2, pp. 107-115.

[4] T. Dörge, J. M. Carstensen, and J. C. Frisvad, "Direct Identification of pure Penicillium species using image analysis," Journal of Microbiological Methods, vol. 41, pp. 121-133, 2000.

[5] J. Kildes $\varnothing$ and B. H. Nielsen, "Exposure assesment of airborne microorganisms by fluorescence microscopy and image processing," Annual Occupational Hygiene, vol. 41, no. 2, pp. 201-216, 1997.
[6] I. M. Inglis and A. J. Gray, "An evaluation of semiautomatic approaches to contour segmentation applied to fungal hyphae," Biometrics, vol. 57, no. 1, no. 232-239, 2001

[7] M.-N. Pons and H. Vivier, "Morphometry of yeast, in: wilkinson m.h.f. and schut f. (eds.)," Digital Image Analysis of Microbes: Imaging, Morphometry, Fluorometry and Motility Techniques and Applications, John Wiley \& Sons Ltd, 1998.

[8] S. Gottwald, C. U. Germeier, and W. Ruhmann, "Computerized image analysis in Fusarium taxonomy," Mycol. Research, vol. 105, no. 2, pp. 206-214.

[9] F. H. L. Benyon, A. S. Jones, E. R. Tovey, and G. Stone, "Differentiation of allergenic fungal spores by image analysis, with application to aerobiological counts," Aerobiologia, vol. 15, pp. 211-223, 1999.

[10] P. Perner, "Why case-based reasoning is attractive for image interpretation," in Proc. International Conference on Case-Based Reasoning, Springer Verlag, 2001, pp. 27-44.

[11] P. Perner, Case-Based Reasoning for Signals and Images, Springer Verlag, 2007.

[12] Umweltanalytik Holbach GmbH. [Online]. Available: http://www.umweltanalytik-holbach.de

[13] P. Perner, T. Günther, H. Perner, G. Fiss, and R. Ernst, "Health monitoring by an image interpretation system-a system for airborne fungi identification," Medical Data Analysis, pp. 64-77, Springer Verlag, 2003.

[14] P. Perner and S. Jänichen, "Recognition of airborne fungi spores in digital microscopic images," Journal of Artificial Intelligence, vol. 36, pp. 137-157, 2006.

[15] I. L. Dryden and K. V. Mardia, Statistical Shape Analysis, John Wiley \& Sons Inc., 1998

[16] S. Jaenichen and P. Perner, "Conceptual clustering and case generalization of two-dimensional forms," Computational Intelligence, vol. 22, pp. 178-193, 2006.

[17] H. Perner and B. Müller, "Mining knowledge for hep-2 cell image classification," Journal of Artificial Intelligence in Medicine, vol. 26, pp. 161-173, 2002.

[18] C.-L. Chang, "Finding prototypes for nearest neighbor classifiers," IEEE Trans. on Computers, pp. 1179-1184, 1974.

[19] P. Perner, Data Mining on Multimedia Data, Springer Verlag, 2002.

[20] D. Wettschereck and D. W. Aha, "Weighting features," Case-Based Reasoning Research and Development, pp. 347-358, Springer-Verlag, Berlin Heidelberg, 1995.

[21] D. W. Aha, D. Kibler, and M. K. Albert, "Instance-based learning algorithm," Machine Learning, vol. 6, pp. 37-66, 1991.

[22] P. Perner, "Prototype-based classification," Applied Intelligence, vol. 28 , no. 3 , pp. $238-246,2008$

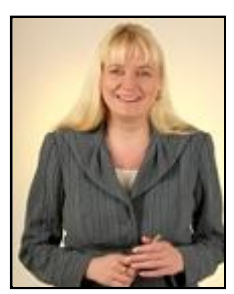

Petra Perner is the director of the Institute of Computer Vision and Applied Computer Sciences IBaI. She received her diploma degree in electrical engineering and her $\mathrm{PhD}$ degree in computer science for the work on "Data Reduction Methods for Industrial Robots with Direct Teach-in-Programing". Her habilitation thesis was entitled "A methodology for the development of knowledge-based image-interpretation systems". She has been the principal investigator of various national and international research projects. She received several research awards for her research work and has been awarded with 3 business awards for her work on bringing intelligent image interpretation methods and data mining methods into business. Her research interest is image analysis and interpretation, machine learning, data mining, big data, machine learning, image mining and case-based reasoning. She was running the Committee TC3 Neuronale Netze and the Technical Committee TC 10 of Machine Learning and Data Mining for IAPR. She is running the Intern. Community of Machine Learning, Data Mining, and Mass Data Analysis of Signals and Images (DSA). Recently, she is working on various medical, chemical and biomedical applications, information management applications, technical diagnosis and e-commerce applications. Most of the developments are protected by legal patent rights and can be licensed to qualified industrial companies. She has published numerous scientific publications and patents and is often requested as a plenary speaker in distinct research fields as well as across disciplines. She is developing efficient data mining and big data methods and parallel computer architectures such as clouds. Her vision is to build intelligent flexible and robust data-interpreting systems that are inspired by the human case-based reasoning process. 